\title{
Force and motion capture system based on distributed micro-accelerometers, gyros, force and tactile sensing
}

\author{
Claudia Latella ${ }^{1}$, Naveen Kuppuswamy ${ }^{1}$ and Francesco Nori ${ }^{1, *}$ \\ ${ }^{1}$ Department of Robotics, Brain, and Cognitive Sciences (RBCS) at Italian Institute of Technology, Via \\ Morego 30, Genova, Italy, (name.surname@ iit.it) \\ * Author to whom correspondence should be addressed; E-Mail: francesco.nori@iit.it.
}

Published: 13 November 2015

\begin{abstract}
Motion capture is a powerful tool used in a large range of applications towards human movement analysis. Although it is a well-established technique, its main limitation is the lack of dynamic information such as forces and torques during the motion capture. In this paper, we present a novel approach for human wearable dynamic (WearDY) motion capture for the simultaneous estimation of whole-body forces along with the motion. Our conceptual framework encompasses traditional passive markers based methods, inertial and contact force sensor modalities and harnesses a probabilistic computational framework for estimating dynamic quantities originally proposed in the domain of humanoid robot control. We present preliminary experimental analysis of our framework on subjects performing a two Degrees-of-Freedom bowing task and we estimate the motion and dynamic quantities. We discuss the implications of our proposal towards the design of a novel wearable force and motion capture suit and its applications.
\end{abstract}

Keywords: wearable dynamics; whole-body estimation; human inverse dynamics.

\section{Introduction}

The importance of knowing dynamic information in human motion, such as forces and torques, is a crucial point in several research areas as ergonomics for industrial scenarios, rehabilitation monitoring or for developing prosthetic devices and exoskeleton systems. Although motion capture is a powerful tool used in a large range of applications towards human movement analysis, its main limitation is the lack of dynamic information as they typically employ only kinematic estimates. For these reasons, human force capture estimation is not a new challenge for the scientific community but the topic has been seldom explored in situ due to the computational difficulties of the analysis. Inspiring from state-of-art approach 
used in solving human inverse dynamics [4][1] and in order to compensate for these computational deadlocks, we propose a novel framework for wearable dynamics (WearDY) by fusing motion and force capture. The approach is computationally based on combining an algorithm to estimate external forces acting on body [3] with a probabilistic method for estimating dynamic quantities in articulated rigid bodies under motion. WearDY architecture will encompass three main modules (Fig. 1): a sensing system comprising accelerometers, gyros, force sensing; a wearable suite where sensor will be properly embedded; a probabilistic algorithm framework to estimate dynamics quantities harnessing redundant measurements information coming from sensors. Basically, the idea is that WearDY architecture will provide a more reliable estimation of forces and motion as the number of sensors increases in this versatile structure: thus the estimation will be more accurate adding information on the human motion.

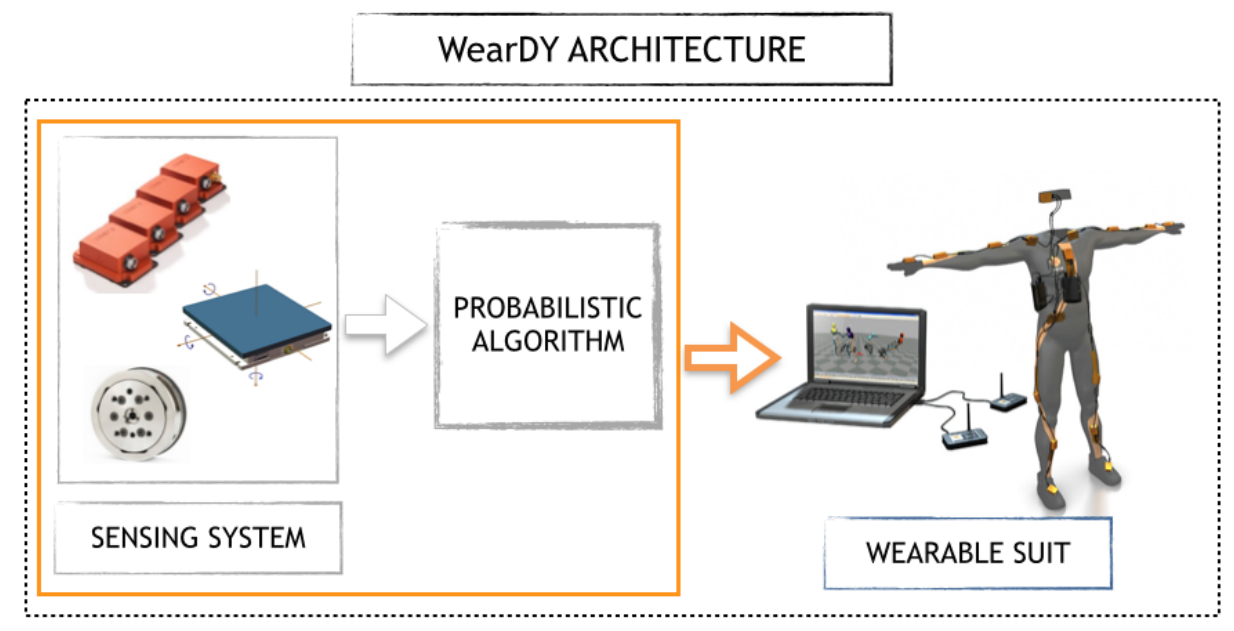

Figure 1. Modules of WearDY architecture.

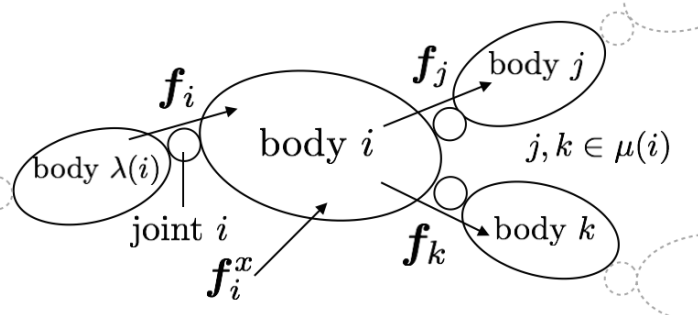

Figure 2. Graphical representation of an articulated rigid body as an oriented kinematic tree.

\section{Methodology}

The notation adopted in WearDY architecture mirrors that in [2]: all variables are spatial vectors (six dimensional vectors including angular quantities in the first three components and the rest as linear quantities). Within this notation, an articulated rigid body system is described as an oriented kinematic tree (Fig. 2) with $n$ Degrees-of-Freedom (DoF) and $N_{B}$ links numbering from 0 (fixed base) to $N_{B}$. Links $i$ and its parent $\lambda(i)$ are coupled with joint $i$ according to Denavit-Hartenberg convention for joint numbering [8]. Joint $i$ motion freedom subspace is modeled with $\boldsymbol{S}_{i} \in \mathbb{R}^{6 \times n_{i}}$, being $n_{i}$ its DoF so $n=n_{1}+\ldots+n_{N_{B}}$. Spatial variables related to link $i$ are defined as follows: velocity $\boldsymbol{v}_{i}$, acceleration $\boldsymbol{a}_{i}$, inertia tensor $\boldsymbol{I}_{i}$, force $\boldsymbol{f}_{i}$ transmitted to body $i$ from $\lambda(i)$, the net force $\boldsymbol{f}_{i}^{B}$ and the external forces 
$\boldsymbol{f}_{i}^{x}$ acting on body $i$. Similarly for joint $i$ are defined the following variables: position $\boldsymbol{q}_{i}$, velocity $\dot{\boldsymbol{q}}_{i}$, acceleration $\ddot{\boldsymbol{q}}_{i}$, spatial velocity across joint $\boldsymbol{v}_{J i}$ and torque $\boldsymbol{\tau}_{i}$.

\subsection{Building Maximum-a-Posteriori dynamics}

The dynamic estimation algorithm has been originally developed in [6] as a framework for the probabilistic estimation of whole-body robot dynamics with redundant measurements. The methodology was here adapted to fit the needs of the human motion. Let us first define a spatial vector $\boldsymbol{d}$ of dynamic variables as follows:

$$
\boldsymbol{d}=\left[\begin{array}{llll}
\boldsymbol{d}_{1}^{\top} & \boldsymbol{d}_{2}^{\top} & \ldots & \boldsymbol{d}_{N_{B}}^{\top}
\end{array}\right]^{\top} \in \mathbb{R}^{18 N_{B}+n}, \quad \boldsymbol{d}_{i}=\left[\begin{array}{lllll}
\boldsymbol{a}_{i}^{\top} & \boldsymbol{f}_{i}^{\top} & \boldsymbol{\tau}_{i} & \boldsymbol{f}_{i}^{x^{\top}} & \ddot{q}_{i}
\end{array}\right]^{\top} \in \mathbb{R}^{18+n_{i}} .
$$

For the physics consistency, $\boldsymbol{d}$ should satisfy a set of constraints deriving from the Newton-Euler equations ${ }^{1}$ for an articulated rigid body, expressed in body $i$ coordinates:

$$
\begin{aligned}
\boldsymbol{a}_{i} & ={ }^{i} \boldsymbol{X}_{\lambda(i)} \boldsymbol{a}_{\lambda(i)}+\boldsymbol{S}_{i} \ddot{\boldsymbol{q}}_{i}+\boldsymbol{v}_{i} \times \boldsymbol{v}_{J i}, \\
\boldsymbol{f}_{i} & =\underbrace{\boldsymbol{I}_{i} \boldsymbol{a}_{i}+\boldsymbol{v}_{i} \times{ }^{*} \boldsymbol{I}_{i} \boldsymbol{v}_{i}}_{\boldsymbol{f}_{i}^{B}}-{ }^{i} \boldsymbol{X}_{0}^{*} \boldsymbol{f}_{i}^{x}+\sum_{j \in \mu(i)}{ }^{i} \boldsymbol{X}_{j}^{*} \boldsymbol{f}_{j} .
\end{aligned}
$$

Given (1), equations (2) can be compactly written in the following matrix equation:

$$
\boldsymbol{D}(\boldsymbol{q}, \dot{\boldsymbol{q}}) \boldsymbol{d}+\boldsymbol{b}_{D}(\boldsymbol{q}, \dot{\boldsymbol{q}})=\mathbf{0},
$$

where $\boldsymbol{D}$ is a block matrix $\in \mathbb{R}^{\left(12 N_{B}+n\right) \times\left(18 N_{B}+2 n\right)}$ and $\boldsymbol{b}_{D}$ is a vector $\in \mathbb{R}^{12 N_{B}+n}$ (see Appendix in [6]). The innovative point is in introducing an explicit equation for measurements by indicating with $\boldsymbol{y} \in \mathbb{R}^{y}$ the value measured by sensors:

$$
\boldsymbol{Y}(\boldsymbol{q}, \dot{\boldsymbol{q}}) \boldsymbol{d}+\boldsymbol{b}_{Y}(\boldsymbol{q}, \dot{\boldsymbol{q}})=\boldsymbol{y}
$$

The structure of $\boldsymbol{Y}$ matrix depends on the number and typology of sensor used for each link. Combining properly (3) and (4), we obtain:

$$
\left[\begin{array}{c}
\boldsymbol{D}(\boldsymbol{q}, \dot{\boldsymbol{q}}) \\
\boldsymbol{Y}(\boldsymbol{q}, \dot{\boldsymbol{q}})
\end{array}\right] \boldsymbol{d}+\left[\begin{array}{l}
\boldsymbol{b}_{D}(\boldsymbol{q}, \dot{\boldsymbol{q}}) \\
\boldsymbol{b}_{Y}(\boldsymbol{q}, \dot{\boldsymbol{q}})
\end{array}\right]=\left[\begin{array}{l}
\mathbf{0} \\
\boldsymbol{y}
\end{array}\right]
$$

Since we consider the incorporation of redundant (and noisy) measurements involved in the analysis, (5) becomes an overdetermined system and an exact solution does not exist. Although approximated solutions can be obtained (least squares or weighted least squares solutions), we move the estimation of $\boldsymbol{d}$ given $\boldsymbol{y}$ in a Gaussian framework by exploiting the sparsity of $[\boldsymbol{D} ; \boldsymbol{Y}]$ matrix by means of Maximum-a-Posteriori (MAP) estimator with the assumption that $\boldsymbol{d}$ and $\boldsymbol{y}$ are stochastic variables with Gaussian distributions ${ }^{2}$ :

$$
\boldsymbol{d}_{\mathrm{MAP}}=\arg \max _{\boldsymbol{d}} p(\boldsymbol{d} \mid \boldsymbol{y})=\arg \max _{\boldsymbol{d}} p(\boldsymbol{d}, \boldsymbol{y})
$$

The primary assumption is to build a joint probability distribution for $\boldsymbol{d}$ and $\boldsymbol{y}$ using the factorization $p(\boldsymbol{d}, \boldsymbol{y})=p(\boldsymbol{d}) p(\boldsymbol{y} \mid \boldsymbol{d})$. The conditioned probability of $\boldsymbol{y}$ given $\boldsymbol{d}$ is $^{3}$ :

1 Assumptions: ${ }^{B} \boldsymbol{X}_{A}$ is the motion vector transformation from $A$ to $B$ coordinates and ${ }^{B} \boldsymbol{X}_{A}^{*}$ is the analogous transformation for a force vector; operator $\times$ is the spatial cross product for motions and its dual $\times^{*}$ is used for forces.

2 By applying Bayes' rule $p(\boldsymbol{d} \mid \boldsymbol{y})=p(\boldsymbol{d}, \boldsymbol{y}) / p(\boldsymbol{y})$, the term $p(\boldsymbol{y})$ can be omitted since it doesn't depend on $\boldsymbol{d}$ and doesn't contribute to the optimization.

3 In the probabilistic notation, given a stochastic variable $\boldsymbol{x}$, with $\boldsymbol{\mu}_{\boldsymbol{x}}$ and $\boldsymbol{\Sigma}_{\boldsymbol{x}}$ it is denoted the mean and covariance of $\boldsymbol{x}$. 


$$
p(\boldsymbol{y} \mid \boldsymbol{d}) \sim \mathcal{N}\left(\boldsymbol{\mu}_{y}, \boldsymbol{\Sigma}_{y}\right), \quad \boldsymbol{\mu}_{y}=\boldsymbol{Y} \boldsymbol{d}+\boldsymbol{b}_{Y} \quad \Rightarrow \quad p(\boldsymbol{y} \mid \boldsymbol{d}) \propto \exp -\frac{1}{2}\left\{\left(\boldsymbol{y}-\boldsymbol{\mu}_{y}\right)^{\top} \boldsymbol{\Sigma}_{y}^{-1}\left(\boldsymbol{y}-\boldsymbol{\mu}_{y}\right\}\right.
$$

The second assumption is to define a probability distribution for prior $p(\boldsymbol{d})$. The best choice leads to:

$$
p(\boldsymbol{d}) \sim \mathcal{N}\left(\overline{\boldsymbol{\mu}}_{D}, \overline{\boldsymbol{\Sigma}}_{D}\right) \quad \Rightarrow \quad p(\boldsymbol{d}) \propto \exp -\frac{1}{2}\left\{\boldsymbol{e}(\boldsymbol{d})^{\top} \boldsymbol{\Sigma}_{D}^{-1} \boldsymbol{e}(\boldsymbol{d})+\left(\boldsymbol{d}-\boldsymbol{\mu}_{d}\right)^{\top} \boldsymbol{\Sigma}_{d}^{-1}\left(\boldsymbol{d}-\boldsymbol{\mu}_{d}\right)\right\}
$$

where the first exponential part represents the contribution of $\boldsymbol{e}(\boldsymbol{d})=\boldsymbol{D} \boldsymbol{d}+\boldsymbol{b}_{D}$ and the second part strictly the prior $p(\boldsymbol{d}) \sim \mathcal{N}\left(\boldsymbol{\mu}_{d}, \boldsymbol{\Sigma}_{d}\right)$. It can be analytically shown that:

$$
\begin{aligned}
& \overline{\boldsymbol{\Sigma}}_{D}=\left(\boldsymbol{D}^{\top} \boldsymbol{\Sigma}_{D}^{-1} \boldsymbol{D}+\boldsymbol{\Sigma}_{d}^{-1}\right)^{-1}, \\
& \overline{\boldsymbol{\mu}}_{D}=\overline{\boldsymbol{\Sigma}}_{D}\left(\boldsymbol{D}^{\top} \boldsymbol{\Sigma}_{D}^{-1} \boldsymbol{b}_{D}-\boldsymbol{\Sigma}_{d}^{-1} \boldsymbol{\mu}_{d}\right) .
\end{aligned}
$$

Given (6) and (7), we can build the conditioned probability $p(\boldsymbol{d} \mid \boldsymbol{y}) \sim \mathcal{N}\left(\boldsymbol{\mu}_{d \mid y}, \boldsymbol{\Sigma}_{d \mid y}\right)$ where

$$
\begin{aligned}
\boldsymbol{\Sigma}_{d \mid y} & =\left(\overline{\boldsymbol{\Sigma}}_{D}^{-1}+\boldsymbol{Y}^{\top} \boldsymbol{\Sigma}_{y}^{-1} \boldsymbol{Y}\right)^{-1}=\left(\boldsymbol{D}^{\top} \boldsymbol{\Sigma}_{D}^{-1} \boldsymbol{D}+\boldsymbol{\Sigma}_{d}^{-1}+\boldsymbol{Y}^{\top} \boldsymbol{\Sigma}_{y}^{-1} \boldsymbol{Y}\right)^{-1} \\
\boldsymbol{\mu}_{d \mid y} & =\boldsymbol{\Sigma}_{d \mid y}\left[\boldsymbol{Y}^{\top} \boldsymbol{\Sigma}_{y}^{-1}\left(\boldsymbol{y}-\boldsymbol{b}_{Y}\right)+\overline{\boldsymbol{\Sigma}}_{D}^{-1} \overline{\boldsymbol{\mu}}_{D}\right] .
\end{aligned}
$$

Since we are interested in the analytic solution of the MAP dynamics (which in the Gaussian case coincides with the minimum variance estimator), if we add multiple statistically independent measurements, $\boldsymbol{y}_{1}=\boldsymbol{Y}_{1} \boldsymbol{d}+\boldsymbol{b}_{Y_{1}}, \ldots, \boldsymbol{y}_{m}=\boldsymbol{Y}_{m} \boldsymbol{d}+\boldsymbol{b}_{Y_{m}}$ in (9a), thus:

$$
\boldsymbol{Y}^{\top} \boldsymbol{\Sigma}_{y}^{-1} \boldsymbol{Y}=\left[\begin{array}{lll}
\boldsymbol{Y}_{1}^{\top} & \ldots & \boldsymbol{Y}_{m}^{\top}
\end{array}\right]\left[\begin{array}{ccc}
\boldsymbol{\Sigma}_{y_{1}}^{-1} & \ldots & 0 \\
\vdots & \ddots & \vdots \\
0 & \ldots & \boldsymbol{\Sigma}_{y_{m}}^{-1}
\end{array}\right]\left[\begin{array}{c}
\boldsymbol{Y}_{1} \\
\vdots \\
\boldsymbol{Y}_{m}
\end{array}\right]=\boldsymbol{Y}_{1}^{\top} \boldsymbol{\Sigma}_{y_{1}}^{-1} \boldsymbol{Y}_{1}+\cdots+\boldsymbol{Y}_{m}^{\top} \boldsymbol{\Sigma}_{y_{m}}^{-1} \boldsymbol{Y}_{m} .
$$

Considering (10), the addition of each measurement induces changes in the associated covariance matrix decreasing at each addition step the uncertainty in the estimation according to:

$$
\boldsymbol{\Sigma}_{d \mid y_{i}}^{-1}=\boldsymbol{\Sigma}_{d \mid y_{i-1}}^{-1}+\boldsymbol{Y}_{i}^{\top} \boldsymbol{\Sigma}_{y_{i}}^{-1} \boldsymbol{Y}_{i}, \quad i=1, \ldots, m
$$

\section{Experimental Section and Theoretical Results}

Experiments have been conducted on three subjects performing a 2-DoF bowing task consisted in three trials each composed of three repetitive bows. Motion data was collected using a Vicon ${ }^{\circledR}$ motion capture system with eight infrared cameras. A total of 14 passive retroreflective markers were properly attached to each participant at key anatomical landmarks in order to capture motion on the sagittal plane. Inspiring by [5], a double-inverted-pendulum (DIP) model and a $\mathrm{URDF}^{4}$ model were developed for each subject with 3 rigid links (foot, leg, torso) and 2 revolute joints for ankle and hip, $\left(N_{B}=2, \boldsymbol{d} \in \mathbb{R}^{40}\right)$. Each subject is asked to perform the task without bending the knee in order to assume leg as a rigid link. No rigid links for head or arms were considered. In addition to the motion capture system, the experimental set-up encompassed an additional sensor (IMU) attached to the torso and a standard AMTI OR6 force platform (Fig. 3). Joint angles $\boldsymbol{q}_{1}$ and $\boldsymbol{q}_{2}$ were computed using only data acquired from the

4 The URDF (Universal Robot Description Format) is a XML-based file format representing a robot model in simulation. 
motion capture system. To evaluated the accuracy of the analysis, we computed the inverse dynamics by using the Newton-Euler algorithm as a future benchmark to validate the MAP method ${ }^{5}$.

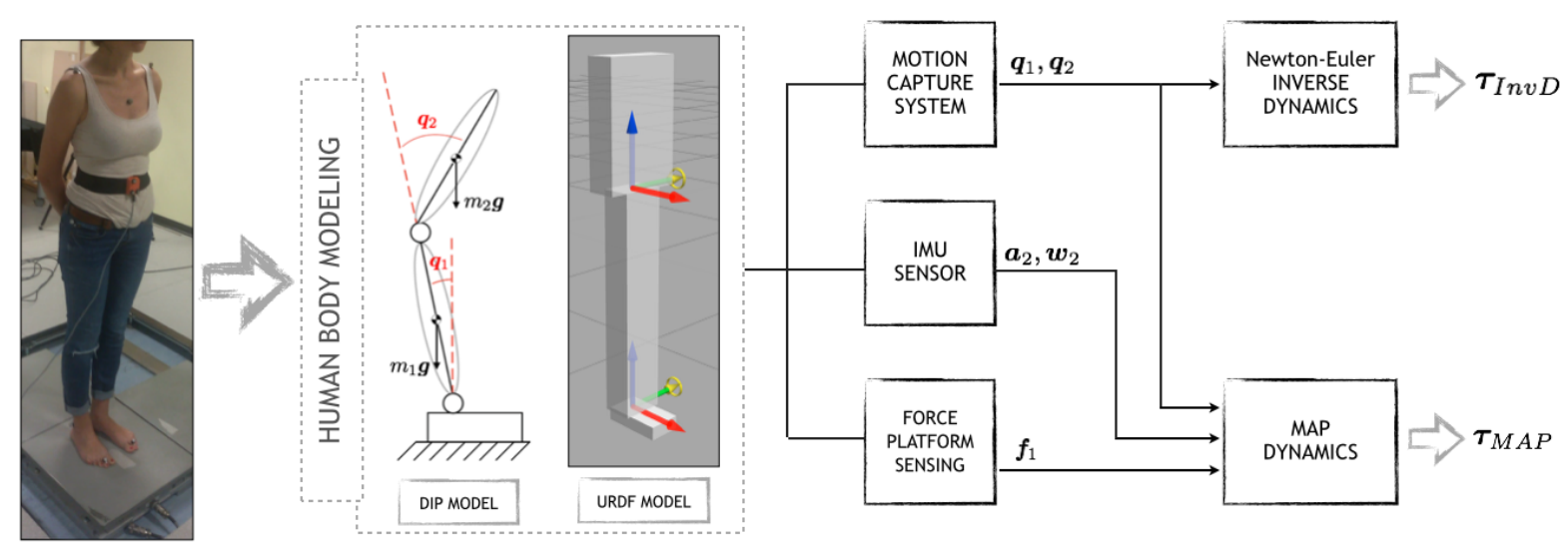

Figure 3. Experimetal set-up for the bowing motion task.
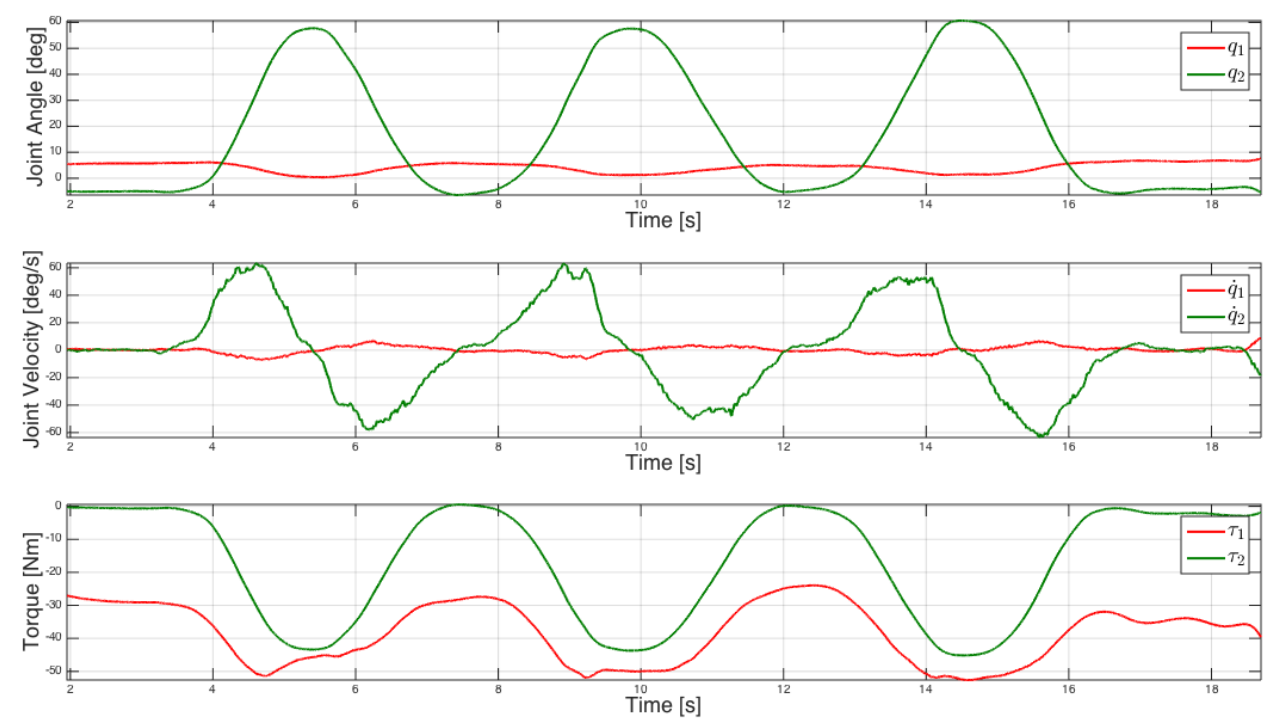

Figure 4. Joint angles $\boldsymbol{q}_{1}$ and $\boldsymbol{q}_{2}$ have been obtained directly from motion capture acquisition; joint velocities have been computed using a third-order polynomial Savitzky-Golay filtering. Joint torques have been computed using a spatial Newton-Euler algorithm approach : it will be used as a benchmark for MAP method validation.

\section{Discussion}

In this paper we presented a theoretical methodology to estimate dynamics quantities along with human motion in order to exploit fusion of sensor information on MAP dynamics framework. Preliminary results in computing Newton-Euler algorithm in the spatial framework provide a reference benchmark to compare forthcoming MAP sensor fusion analysis. Since WearDY is still a conceptual prototype, future works will be aimed at real-time implementation, at the actual design of the wearable garment and in the improvement of the sensor architecture in order to make the system reliable in

5 To test benchmark robustness we performed inverse dynamics on two different libraries: a Matlab ${ }^{\circledR}$ library implemented in [2] and a C++ library [7] (with a comparison error of $10^{-12}$ ). 
situ. Computationally, it will be fundamental integrate MAP dynamics with a state estimator such as an Extended Kalman filter combining obtained a-posteriori estimate with a-priori estimate of the filter state. From a design perspective, the wearable system will comprise of a soft sensing garment with embedded sensors that facilitate free movement of a human subject. The suit design will ideally exploit the material compliance in order to improve sensor reliability through elimination of the error introduced by the interface between the garment and the subject's skin. Another important future objective concerns the possibility of integrating with the existing set of sensors, an electromyography (EMG) analysis in order to provide information on muscles activity. A realistic scenario of suit application should be the rehabilitation framework wherein a wearable garment will be necessary for monitoring patients or to create more ergonomics and compliant prosthesis and exoskeleton systems. The proposed suit and the forthcoming improvements would permit substantial enhancements in the analysis of human movements in motion scenarios.

\section{Acknowledgments}

This paper was supported by the FP7 EU projects CoDyCo (No. 600716 ICT 2011.2.1 Cognitive Systems and Robotics), and Koroibot (No. 611909 ICT-2013.2.1 Cognitive Systems and Robotics)

\section{Conflicts of Interest}

The authors declare no conflict of interest.

\section{References}

1. V. Cahouet, M. Luc, and D. Amarantini. Static optimal estimation of join accelerations for inverse dynamics problem solution. Journal of Biomechanics, 35, pages 1507-1513, 2002.

2. R. Featherstone. Rigid Body Dynamics Algorithms. Springer, 2008.

3. M. Fumagalli, S. Ivaldi, M. Randazzo, L. Natale, G Metta, G. Sandini, and F. Nori. Force feedback exploiting tactile and proximal force/torque sensing. Autonomous Robots, 33(4):381-398, 2012.

4. K. Guelton, S. Delprat, and T-M Guerra. An alternative to inverse dynamics joint torques estimation in human stance based on takagi-sugeno unknown-inputs observer in the descriptor form. Control Engineering Practice, 16, pages 1414-1426, 2008.

5. G. Hettich, L. Fennel, and T. Mergner. Double inverted pendulum model of reactive human stance control. Multibody Dynamics 2011, ECCOMAS Thematic Conference, 2011.

6. F. Nori, N. Kuppuswamy, and S. Traversaro. Simultaneous state and dynamics estimation in articulated structures. In Intelligent Robots and Systems (IROS), 2015 IEEE/RSJ International Conference on. [Accepted for publication], 2015.

7. F. Nori, S. Traversaro, J. Eljaik, F. Romano, A. Del Prete, and D. Pucci. icub whole-body control through force regulation on rigid noncoplanar contacts. Frontiers in Robotics and AI, 2(6), 2015.

8. B. Siciliano, L. Sciavicco, L. Villani, and G. Oriolo. Robotics: Modelling, Planning and Control. Advanced Textbooks in Control and Signal Processing. Springer, 2009.

(c) 2015 by the authors; licensee MDPI, Basel, Switzerland. This article is an open access article distributed under the terms and conditions of the Creative Commons Attribution license (http://creativecommons.org/licenses/by/4.0/). 